\title{
Alters- und alternsgerechte Arbeitsgestaltung - Offene Fragen für Wissenschaft und betriebliche Praxis
}

\section{Müblenbrock}

\section{Abstract}

Die Berücksichtigung von Altersverläufen in der Arbeitsgestaltung hat in den vergangenen 20 Jahren im Bereich der Forschung deutlich zugenommen. Modelle zum Job Design sind weiterentwickelt und mit Alterstheorien kombiniert worden, um die Regulation von Tätigkeiten über die Lebensspanne hinweg zu verstehen. Das Kapitel differenziert zwischen alterns- und altersgerechter Arbeitsgestaltung und beschreibt vier zentrale Ansatzpunkte (Arbeitsaufgabe, -organisation, soziale Beziehungen und Arbeitsumfeld). Abschließend werden Herausforderungen für die Forschung (z.B. Erforschung praxisnaher Instrumente) und für die Praxis (z.B. konsequente Umsetzung der Gefährdungsbeurteilung) dargestellt.

\section{Einleitung}

Dass Arbeitsgestaltung eine zentrale Determinante für den Erhalt der Erwerbsfähigkeit ist, ist seit den 1960er-Jahren systematisch bekannt. In der deutschsprachigen Arbeitspsychologie hat lange Zeit die Handlungsregulationstheorie (u.a. Hacker, 1998) dominiert, die insbesondere die persönlichkeitsfördernde Gestaltung von Arbeitssystemen als Ziel hatte. Auf dem Kongress „Altern und Arbeit" vor 20 Jahren stand eben jene Theorie im Mittelpunkt der Arbeitsgruppe ,Arbeitsgestaltung'. Ergebnis der erfolgreichen Zusammenarbeit von Wissenschaft (Fraunhofer Institut) und Praxis (ehemals DaimlerChrysler AG) war die Kongress-Präsentation eines Systems zur Analyse, Bewertung und Gestaltung von Arbeitstätigkeiten, das forschungsbasiert ausgearbeitet und in der Industrie eingesetzt wurde (s. von Rothkirch, 2000). Ziel des eingesetzten Tätigkeitsbewertungssystems (Hacker \& Richter, 1980) ist es, vielfältig wechselnde psychische Anforderungen und vollständige (im Sinn von Ganzheitlichkeit) Tätigkeiten zu generieren, z.B. durch die Steuerung von Denkanforderungen oder Lernanreizen. Für eine allgemein humane Arbeitssystemgestaltung haben Tätig- 


\section{Müblenbrock}

keitsbewertungssysteme einen profunden Beitrag geleistet, einen konkreten Bezug zum Altern lieferten sie jedoch noch nicht. So merkte Prof. Wachtler in der Zusammenfassung seiner Arbeitsgruppe im Jahr 2000 kritisch an: „Formen der Neugestaltung von Arbeit [werden] in den Unternehmen relativ unabhängig von demographischen Prozessen durchgeführt werden. [...] es sieht nicht so aus, als ob in den Unternehmen bereits tiefgreifende Restrukturierungs- und Umgestaltungsprozesse im Gang wären, um den mit dem demographischen Wandel verbundenen Problemen gerecht zu werden“" (von Rothkirch, 2000, S. 429).

\section{Forschung zur Arbeitsgestaltung im Wandel}

Die Berücksichtigung von Altersverläufen bei der Arbeitsgestaltung hat sich in den vergangenen 20 Jahren - zumindest für den Bereich der Forschung - deutlich verändert. Modelle und Theorien zum Job-Design sind weiterentwickelt worden, wurden verfeinert und mit Alterstheorien kombiniert, um die Regulation von Tätigkeiten über die Lebensspanne hinweg zu verstehen. Tabelle 1 skizziert die zentralen Theorien, die einflussreich für Forschung und unternehmerische Praxis in den vergangenen Jahren waren.

Tabelle 1: Wichtige theoretische Ansätze der Arbeitspsychologie und Alternsforschung (u.a. Hertel \& Zacher, 2018)

\begin{tabular}{|l|l|}
\hline Theoretische Ansätze & Zentrale Erkenntnisse \\
\hline $\begin{array}{l}\text { Job Demands-Re- } \\
\text { sources Modell } \\
\text { (Bakker \& Demerouti, } \\
\text { 2007) }\end{array}$ & $\begin{array}{l}\text { Arbeitsbedingungen lassen sich in Anforderungen } \\
\text { (z.B. psychisch oder physisch) und Ressourcen (z.B. } \\
\text { Autonomie) einteilen. Während Arbeitsanforderun- } \\
\text { gen die Gesundheit negativ beeinflussen (z.B. Er- } \\
\text { schöpfung), wirken Arbeitsressourcen über einen } \\
\text { motivationalen Prozess auf Arbeitsengagement oder } \\
\text {-motivation positiv. Diese Prozesse sind nicht unab- } \\
\text { hängig voneinander, sondern interagieren. Das Mo- } \\
\text { dell erklärt z.B. die kompensatorische Wirkung von } \\
\text { sozialer Unterstützung bei hohen Arbeitsanforde- } \\
\text { rungen auf Erschöpfung. Job Crafting ist als Ele- } \\
\text { ment im Wirkprozess integriert. }\end{array}$ \\
\hline
\end{tabular}




\begin{tabular}{|l|l|}
\hline $\begin{array}{l}\text { (Motivationale) Theo- } \\
\text { rie der Entwicklung } \\
\text { über die Lebensspan- } \\
\text { ne (Baltes, 1987; } \\
\text { Heckhausen, Wrosch } \\
\text { \& Schulz, 2010) }\end{array}$ & $\begin{array}{l}\text { forderungen. Dies erfordert Lernen und Anpas- } \\
\text { sung sowie die Notwendigkeit, Ressourcen effek- } \\
\text { tiv einzusetzen. Motive und Selbstregulation am } \\
\text { Arbeitsplatz unterliegen altersabhängigen Verän- } \\
\text { derungen. Das Motiv der Generativität (Hilfeleis- } \\
\text { tung, Weitergabe an andere Generationen) ist bei } \\
\text { äleren Beschäftigten am stärksten ausgeprägt. }\end{array}$ \\
\hline $\begin{array}{l}\text { SOC-Modell (Selekti- } \\
\text { on, Optimierung, } \\
\text { Kompensation; Baltes } \\
\text { \& Baltes, 1990) }\end{array}$ & $\begin{array}{l}\text { Um abnehmende Ressourcen und Fähigkeiten } \\
\text { mit zunehmendem Alter zu bewältigen, sind älte- } \\
\text { re Beschäftigte in der Lage, entsprechende Strate- } \\
\text { gien bewusst einzusetzen. Unternehmen setzen } \\
\text { SOC-Trainings ein, um die Arbeitsfähigkeit der } \\
\text { Beschäftigten zu stärken. }\end{array}$ \\
\hline $\begin{array}{l}\text { Sozio-emotionale Se- } \\
\text { lektivitäts-Theorie } \\
\text { (SST; Carstensen, } \\
\text { 2006) }\end{array}$ & $\begin{array}{l}\text { Nicht das kalendarische Alter ist zentral für das } \\
\text { Setzen individueller Ziele und Handlungen, son- } \\
\text { dern die wahrgenommene verbleibende Zeit } \\
\text { (,Future Time Perspective', FTP). Bei scheinbar } \\
\text { unbegrenzter FTP (d.h. eher jüngere Beschäftig- } \\
\text { te), überwiegen die Ziele Informationserwerb } \\
\text { und Erweiterung sozialer Netzwerke. Bei be- } \\
\text { grenzter FTP (d.h. eher ältere Beschäftigte) ste- } \\
\text { hen Gefühle, emotionales Wohlbefinden und die } \\
\text { Gegenwart im Fokus des Handelns. Die Theorie } \\
\text { erklärt die altersabhänge Veränderung von Moti- } \\
\text { ven und Arbeitseinstellungen und warum eine } \\
\text { Passung zwischen Motiven und Arbeitsmerkma- } \\
\text { len für ältere Beschäftigte wichtiger ist als für jün- } \\
\text { gere. }\end{array}$ \\
\hline
\end{tabular}

Die Konsequenzen des demografischen Wandels, d.h. ein wachsendes Durchschnittsalter der Belegschaften, eine zunehmende Altersdiversität oder der spürbare Fachkräftemangel, haben die Investitionen in Forschungs- und Förderprogramme gerade zu Beginn der 2000er-Jahre verstärkt. Wichtig und folgerichtig war die Kombination von Theorien der Arbeitsgestaltung mit Alterstheorien bzw. Theorien der Lebensspanne. Für die Forschung und Anwendung bedeutete dies die wichtige Erkenntnis, zeitliche Verläufe (z.B. Alterungsprozesse) zu berücksichtigen, da Merkmale der Arbeitsgestaltung nicht statisch, sondern in Abhängigkeit von veränderten arbeitsbezogenen und individuellen Merkmalen (z.B. Betriebskli- 


\section{Müblenbrock}

ma, Alter) dynamisch wirken. In ihrem Rahmenkonzept zur Erklärung von Stress, Emotionsregulation und Wohlbefinden über die Lebensspanne haben z.B. Scheibe und Zacher (2013) dies konsequent umgesetzt. So leiten sie u.a. ab, dass zwischenmenschliche Konflikte besonders jüngere Beschäftigte beanspruchen, Work-Life-Konflikte und starke arbeitsbezogene Verantwortung besonders Beschäftigte mittleren Alters beeinträchtigen und eine Vermeidung von negativem oder stark emotionalem Erleben am Arbeitsplatz bei älteren Beschäftigten stattfindet.

\section{Differenzierung zwischen alterns- und altersgerechter Arbeitsgestaltung}

Entsprechend differenzierter Erkenntnisse zur Wirkung von Arbeitsgestaltung und der Rolle von Alter ist eine Unterscheidung zwischen alters- und alternsgerechter Gestaltung von Arbeit sinnvoll. Gut gestaltete Arbeit ist gerade für ältere Arbeitnehmerinnen und Arbeitnehmer wichtig, da sie möglicherweise ungünstige Arbeitsbedingungen (z.B. körperliche schwere Arbeit) über einen längeren Zeitraum kumuliert erlebt haben und sich zudem negative Effekte in Kombination mit natürlichen Alterungsprozessen (z.B. reduzierte Muskelkraft) verstärken können. Eine altersgerechte Arbeitsgestaltung ermöglicht es, Beschäftigungspotenziale auszuschöpfen, indem Bedarfe und Fähigkeiten bestimmter Altersgruppen in den Fokus gerückt werden. Die Sichtweise ist kompensatorisch und lebensphasenorientiert. Aufgrund des zunehmenden Durchschnittsalters in den Belegschaften stehen gegenwärtig vor allem ältere Beschäftigte im Fokus einer altersgerechten Arbeitsgestaltung (z.B. altersabhängige Reduzierung von Nachtschichten). Zudem nehmen prospektive und präventive Strategien die heute jüngeren und mittelalten Beschäftigten in den Blick. Eine alternsgerechte Arbeitsgestaltung betrachtet entsprechend allgemeine Prinzipien gut gestalteter Arbeit über das gesamte Erwerbsleben, damit Beschäftigte möglichst lange gesund, motiviert und leistungsfähig erwerbstätig sein können. Die Sichtweise ist hier eine eher primärpräventive und zukunftsorientierte (BAuA, 2017).

Empirisch gesicherte Erkenntnisse im Hinblick auf Altersunterschiede sind beispielsweise, dass körperliche und physiologische Fähigkeiten (z.B. sensorisch, kardiovaskulär, neurologisch) mit dem Alter durchschnittlich abnehmen. Dabei sind die interindividuellen Unterschiede teils erheblich. Auch haben jüngere Beschäftigte im Vergleich zu älteren eine höhere fluide Intelligenz und eine durchschnittlich höhere Lernmotivation. Ältere Mitarbeiterinnen und Mitarbeiter weisen hingegen höhere Fähigkeiten der emotionalen und Selbst-Regulation, mehr aktive Bewältigungsstrategien, 
eine höhere Arbeitszufriedenheit oder ein höheres Bedürfnis nach Autonomie und Generativität auf (s. u.a. Hertel \& Zacher, 2014).

$\mathrm{Zu}$ berücksichtigen ist jedoch, dass diese und weitere altersabhängige Entwicklungen im jeweiligen (Arbeits-)Kontext stattfinden, sodass Entwicklungen bestimmter Fähigkeiten nicht nur auf das biologische Altern zurückzuführen sind, sondern auch langfristige Einflüsse belastender bzw. förderlicher Arbeitsbedingungen deutlich werden. So zeigt sich entsprechend, dass das Risiko schlechter Gesundheit ungleich zwischen Beschäftigten verschiedener Berufsgruppen verteilt ist (Hasselhorn, 2015).

\section{Ansatzpunkte alterns- und altersgerechter Arbeitsgestaltung}

Verschiedene Übersichtarbeiten und Meta-Analysen haben in den vergangenen Jahren den Blick auf die Rolle des Alters geschärft und damit auf Ansatzpunkte einer altersgerechten Arbeitsgestaltung (Zacher \& Schmitt, 2016; Truxillo, Cadiz \& Hammer, 2015; Mühlenbrock \& Hüffmeier, 2020; Bögel \& Frerichs, 2011; Gerst, 2013). Dabei steht konzeptionell die Frage im Vordergrund, inwiefern Alter den Zusammenhang zwischen Arbeitsbedingungen und arbeitsbezogenen Merkmalen moderiert (s. Abb. 1). Hinsichtlich einer gesundheitsförderlichen und alternsgerechten Arbeitsgestaltung lassen sich Arbeitsbedingungen in vier Merkmalsbereiche unterscheiden (Beck et al., 2017; BAuA, 2017)1. 
Abbildung 1: Rolle des Alter $(n)$ s im Kontext der Arbeitsgestaltung

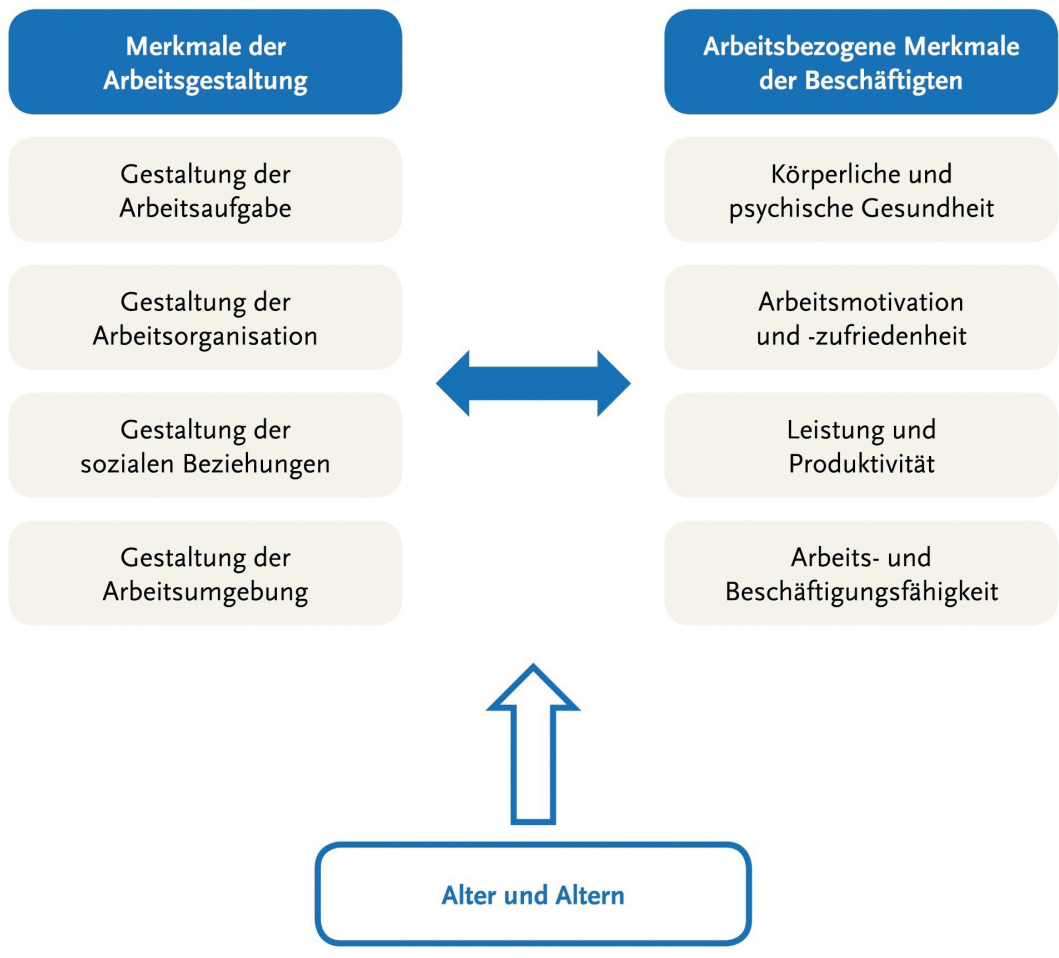

Untersuchungsmodell der alter(n)sgerechten Arbeitsgestaltung

\subsection{Arbeitsaufgabe}

Für den Erhalt der kognitiven Leistungsfähigkeit, Arbeitsmotivation und -zufriedenheit sind Arbeitsaufgaben elementar, die angemessen fordern und fördern. Handlungsspielräume sind wichtig aufgrund ihrer positiven Wirkungen auf die Leistungsfähigkeit und Arbeitsmotivation und daher ein zentrales Merkmal einer alternsgerechten Arbeitsgestaltung. Mit dem Alter gewinnt dies an Bedeutung, da es Beschäftigten eine größere Autonomie ermöglicht, ihre Stärken besser einzubringen und altersbedingte Einschränkungen zu kompensieren (Baltes \& Baltes, 1990). Abwechslungsreiche Aufgaben vermeiden zudem einseitige Anforderungen und erweitern das Fähigkeitsprofil und Einsatzmöglichkeiten. Aufgabenvielfalt wirkt sich 
positiv auf Arbeitsmotivation, -zufriedenheit und -leistung aus, bei jüngeren Beschäftigten stärker als bei älteren. Ältere Beschäftigte profitieren hingegen stärker als jüngere von einer Anforderungsvielfalt. Kognitiv fordernde Tätigkeiten können die intellektuelle Flexibilität erhalten und den altersbedingten kognitiven Leistungsabfall reduzieren. Gestaltungsmöglichkeiten wie z. B. Job Enlargement, Job Enrichment oder Job Rotation tragen zum Abwechslungsreichtum bei. Aufgabenkomplexität beeinflusst ebenfalls die kognitive Leistungsfähigkeit. Bei Beschäftigten, die in einem sehr gering komplexen und unterfordernden Arbeitsumfeld arbeiten, verschlechtert sich die kognitive Leistungsfähigkeit schneller als vergleichsweise zu denjenigen, die in einem mental anregenden Umfeld tätig sind (Oltmanns et. al 2017a). Mit zunehmendem Alter verstärkt sich dieser Zusammenhang. Entsprechend steigt auch die Bedeutung einer lernförderlichen Aufgabengestaltung oder zumindest von Tätigkeitswechseln (Oltmanns et al 2017b, Godde et al 2019). Im Umgang mit emotionalen Anforderungen zeigen sich jedoch Ältere mehrheitlich im Vorteil gegenüber Jüngeren: Ältere setzen Bewältigungsstrategien effektiver ein und fühlen sich weniger emotional erschöpft. Durch ihre Berufserfahrung und ihre emotional-sozialen Kompetenzen zeigen sich ältere Beschäftigte zudem häufig flexibler in der Anwendung angemessener Strategien in emotional anspruchsvollen Situationen. Daher sollten Maßnahmen zur Förderung der Emotionsarbeit besonders bei jüngeren Beschäftigten ansetzen.

\subsection{Arbeitsorganisation}

Die Gestaltung der Arbeitsorganisation lässt sich hinsichtlich Arbeitszeit und Arbeitsabläufe unterscheiden. Wichtig ist bei der Arbeitszeitgestaltung zu berücksichtigen, dass sich die Belastbarkeit der Beschäftigten mit dem Alter verändert. So ist die persönliche Beeinträchtigung durch überlange Arbeitszeiten aufgrund von Vorbelastungen im fortgeschrittenen Lebensalter häufig höher als in jüngeren Jahren. Ein rechtzeitiger Ausgleich von Arbeitszeitbelastungen und ausreichenden Erholungszeiten ist deshalb zu berücksichtigen. Dabei gilt, dass eine Entlastung Älterer nicht zu einer Überbelastung Jüngerer führen sollte. Gestaltungsmöglichkeiten betreffen hier beispielsweise Pausen, Ruf-, Nacht- und Schichtdienst (Brenscheidt, 2017). Dauernachtarbeit sollte vermieden und Nachtschichten so weit wie möglich ausgedünnt und arbeitswissenschaftliche Erkenntnisse eingeplant werden. Studienergebnisse zeigen mehrheitlich, dass die gesundheitliche Toleranz für Schichtarbeit mit dem Alter abnimmt. Ebenfalls erleichtern lebensphasenorientierte Arbeitszeitmodelle wie z.B. Telearbeit, Gleit- oder 


\section{Müblenbrock}

Teilzeitmodelle die Vereinbarkeit von beruflichen und privaten Verpflichtungen oder Interessen. Dazu gehört auch ein gleitender Ausstieg aus dem Erwerbsleben. Auch die Art des Arbeitsablaufs kann zur Überforderung der Beschäftigten führen. Ursachen dafür können vielfältig sein wie z.B. hohe Arbeitsintensität oder häufige Arbeitsunterbrechungen. Zusammenhänge zwischen hoher Arbeitsintensität und schlechter körperlicher sowie psychischer Gesundheit sind vielfach belegt. Im Sinne einer alter(n)sgerechten Arbeitsgestaltung empfiehlt es sich, Arbeitsgeschwindigkeiten generell an individuelle Voraussetzungen anzupassen. Arbeitsunterbrechungen gehören zu den zentralen Stressoren in der Arbeitsorganisation und stellen hohe Anforderungen an das Arbeitsgedächtnis und die Aufmerksamkeit. Sie sind mit Leistungseinbußen und zunehmender Erschöpfung verbunden. Obwohl sich kognitive Prozesse mit dem Alter verschlechtern können, ermöglichen Berufserfahrung im Umgang mit Arbeitsunterbrechungen und die damit oft verbundene Gelassenheit gegenüber Anforderungen des Berufsalltages eine ausgleichende Wirkung. Ergänzend tragen systematisch eingesetzte Belastungs- und Tätigkeitswechsel zu einer alter $(\mathrm{n})$ sgerechten Arbeitsorganisation bei. Arbeits- und Beschäftigungsfähigkeit werden gestärkt.

\subsection{Soziale Beziehungen}

Soziale Beziehungen, Interaktionen und Zusammenarbeit sind wichtige Bestandteile des Arbeitsprozesses. Das soziale Arbeitsumfeld wirkt einerseits durch soziale Unterstützung von Kolleginnen bzw. Kollegen und Führungskräften förderlich auf Zufriedenheit, Gesundheit und Leistung der Beschäftigten. Andererseits ist ein schlechtes Betriebs- oder Gruppenklima, insbesondere Konflikte am Arbeitsplatz, belastend und stressauslösend. Fehlt die soziale Unterstützung, wirkt sich dies negativ z. B. auf die emotionale Erschöpfung und das Wohlergehen aus. Das gilt für Ältere stärker als für Jüngere. Ein respektvolles und wertschätzendes Miteinander ist auch ein wesentlicher Faktor dafür, möglichst lang im Erwerbsleben bleiben zu wollen. Eine Führung, die die Bedarfe bestimmter Altersgruppen berücksichtigt (altersgerechte Führung) wirkt sich gesundheitsförderlich aus (Jungmann, Bilinska \& Wegge, 2014). 


\subsection{Arbeitsumgebung}

Der Faktor „Arbeitsumgebung“ umfasst am ehesten Aspekte des klassischen Arbeitsschutzes. Hier stehen Veränderungen der körperlichen Leistungsfähigkeit über die Altersspanne im Vordergrund. Diese betreffen in erster Linie sensorische und muskuläre Einschränkungen sowie Veränderungen des Herz-Kreislauf-Systems (Keil u.a. 2017). Die in diesen Bereichen auftretenden Alterseffekte sind durch technische, organisatorische und persönliche Maßnahmen jedoch oftmals zu kompensieren. Die altersbedingte Verschlechterung der Seh- und Hörfähigkeit kann durch gezielte Gestaltung ausgeglichen oder abgeschwächt werden, z.B. durch höhere Beleuchtungsstärken und Kontraste, weniger Blendung und größere Anzeigen (Soestmeyer, 2017). Die Reduktion von Hintergrundgeräuschen und dauerhaftem Lärm, die Erhöhung der Eindeutigkeit von Signalen oder die Nutzung von persönlichem Gehörschutz in Lärmbereichen sind ebenfalls förderlich (Schlittmeyer, 2017). Mit dem Alter steigt die Beanspruchung unter extremen klimatischen Bedingungen (Hitze, Kälte, Feuchtigkeit, Zugluft). Diese sollten daher bei älteren Beschäftigten möglichst vermieden werden. Arbeitsorganisatorische Maßnahmen wie etwa kürzere Einsatzzeiten durch Pausen oder Rotationen reduzieren gesundheitliche Belastungen. Bezüglich arbeitsbedingter Vibrationen verstärken altersbedingte Erkrankungen ihre negativen Effekte auf die Gesundheit, so dass eine Reduzierung von Vibrationen mit dem Alter wichtiger für die Gesunderhaltung wird. Körperliche Erholung ist für den Erhalt der Leistungsfähigkeit und zur Vorbeugung von Verletzungen und Erschöpfung von großer Bedeutung (Wendsche \& Lohmann-Haislah, 2018): Bei gleichen Belastungen ist es mit zunehmendem Alter wahrscheinlicher, an der eigenen Leistungsgrenze zu arbeiten. Eine systematische Nutzung von Hilfsmitteln, die Erhöhung von Autonomie und Partizipation sowie systematische Tätigkeitswechsel verringern die negativen Auswirkungen. Ergonomisch ungünstige Haltungen und Tätigkeiten sollten daher verringert, besser noch vermieden werden z.B. durch die Vermeidung von Zwangshaltungen und übermäßigem Beugen, Bücken und Verdrehen, um arbeitsbezogene MuskelSkelett-Erkrankungen und Sturzgefahren zu reduzieren. Arbeitsmittel und Technologien sollten (unabhängig vom Alter) an die körperlichen Eigenschaften und Kompetenzen der Beschäftigten angepasst sein. Maßnahmen für eine ergonomisch gut gestaltete Arbeitsumgebung sind daher auch Maßnahmen einer alternsgerechten Arbeitsgestaltung. 


\section{Müblenbrock}

\section{Herausforderungen für Forschung und Praxis im Themenfeld Arbeitsgestaltung}

\subsection{Herausforderungen für die Forschung}

Eine zentrale Herausforderung für die Zukunft ist die Entwicklung bzw. Anwendung integrativer Theorien und eine entsprechende spezifische empirische Überprüfung dessen. Die theoretischen Rahmen zur Ableitung altersgerechter Arbeitsgestaltung sind in den vergangenen Jahren zwar fortgeschritten, integrative, verifizierte Theorien zum erfolgreichen Altern am Arbeitsplatz (,successful aging') fehlen noch. Aktivitäten der internationalen Arbeitsgruppe um Hannes Zacher sind allerdings vielversprechend. Sie fokussieren Theorien zur Lebensspanne im Arbeitskontext (Rudolph, Zacher \& Baltes, 2019) und verknüpfen diese z.B. mit der Handlungsregulationstheorie in einer ,Metatheorie' (ARAL, s. Zacher, Hacker \& Frese, 2016). Diese versucht zu erklären, wie Beschäftigte ihr (Arbeits-)Umfeld über die Erwerbsspanne beeinflussen und von ihr beeinflusst werden. Für die Ableitung konkreter, praxisnaher Empfehlungen stehen dazu aber noch ausreichende empirische Untersuchungen aus. Generell stellt sich bei der Erforschung von Arbeitsgestaltungsparametern die Frage, welches Forschungsdesign die ,besten' Erkenntnisse liefert. So ermöglicht die Untersuchung singulärer Merkmale (z.B. Anforderungsvielfalt) zwar eine größere Klarheit zu dessen Effekten im Kontext von Alter (s. Mühlenbrock \& Hüffmeier, 2020), allerdings wirken diese meistens in Abhängigkeit vieler Variablen der organisationalen Kontexte.

Vielversprechend für die Arbeitsgestaltung sind Forschungsergebnisse der vergangenen zehn Jahren zu proaktiven, von den Beschäftigten selbst initiierten Gestaltungsstrategien. Job Crafting ermöglicht den Mitarbeiterinnen und Mitarbeitern, die Person-Job-Passung zu erhöhen, indem aufgabenbezogene, soziale und kognitive Elemente der Tätigkeit gestaltet werden (z.B. Feedback einholen, positives Erleben stärken). Job Crafting hat sich als Faktor für ,successful aging' herausgestellt und kann insbesondere für ältere Beschäftigte wichtig und vorteilhaft sein (Wong \& Tetrick, 2017).

Nicht nur das kalendarische Alter, sondern auch damit verbundene Konstrukte sollten in die Betrachtung von Alterung am Arbeitsplatz einbezogen werden, da die Einflussfaktoren und Lebensverläufe mit zunehmendem Alter diverser werden und damit die Vorhersagekraft des kalendarischen Alters abnimmt. Als relevante Operationalisierungen haben sich z.B. FTP (Future Time Perspective, s.o.), funktionales Alter (z.B. Gesundheit), lebensspannenbezogenes Alter (z.B. Lebenssituation), psychosoziales Alter 
(z.B. soziale bzw. Selbstwahrnehmung) oder organisationales Alter (z.B. Betriebszugehörigkeit) herausgestellt (de Lange et al., 2006). Damit wird deutlich, dass das kalendarische Alter als alleinige Erklärungsvariable nicht dienlich ist und andere personenbezogene Variablen, wie Gender oder Persönlichkeit nicht irrelevant zu sein scheinen (Oldham \& Fried, 2016). Des Weiteren verlaufen die Zusammenhänge zwischen Alter und arbeitsbezogenen Variablen (z.B. Leistung oder Motivation) nicht zwangsläufig linear. Eine Prüfung kurvilinearer Zusammenhänge ist notwendig, um z.B. die Rolle des mittleren Beschäftigtenalters genauer verstehen zu können (z.B. Mühlenbrock \& Hüffmeier, 2020). Außerdem sollten hinsichtlich Aussagekraft und Ursachenforschung Studien längsschnittlich angelegt sein weniger um „das Alter“, sondern um Alternsprozesse zu erforschen. Längsschnittstudien können zudem Kohorten- oder Selektionseffekte identifizieren.

Zentral für eine tatsächliche Umsetzung der arbeitsgestalterischen Erkenntnisse in die Praxis ist jedoch die Erforschung praxisnaher Instrumente und Prozesse, um den ,Knowing-Doing-Gap' zu reduzieren (s. Langhoff in diesem Band; Ilmarinen, 2017). Was behindert z.B. eine Umsetzung alternsgerechter Arbeitsgestaltung in der Praxis? Wie ist diesen Hindernissen effektiv zu begegnen? Welche Argumente und Fähigkeiten benötigen die für die Umsetzung zuständigen Akteure?

\subsection{Praxisbezogene Herausforderungen im Themenfeld "Arbeitsgestaltung“}

Alternssensible Arbeitsgestaltung ist wirksamer und gezielt umzusetzen, wenn ihre Implementierung systematisch erfolgt. Idealerweise sollten Maßnahmen in bestehende Strukturen zur Förderung der Beschäftigtengesundheit eingebettet sein und es sollte ein in den relevanten Bereichen abgestimmtes Vorgehen existieren, z.B. zwischen Arbeitssicherheit, Arbeitsmedizin, betrieblichem Gesundheitsmanagement, Personal und Führung. Für die Ableitung konkreter Ansatzpunkte zur Gestaltung von Arbeitsbedingungen ist die (psychische) Gefährdungsbeurteilung das wesentliche Instrument (BAuA, 2016). Hinsichtlich des Personalmanagements sollte dies alterssensibel ausgerichtet sein und z.B. Laufbahnen und Lebensphasen bei Maßnahmen berücksichtigen, um Potenziale zu erhalten und Ressourcen zu entwickeln (Sonntag \& Seiferling, 2017). Eine wertschätzende und faire Unternehmens- und Führungskultur kann darüber hinaus zu einem alternsgerechten Klima beitragen, das z.B. nicht altersdiskriminierend ist, sondern die Stärken jedes einzelnen Beschäftigten fördert (Jungmann et al., 2014). 


\section{Müblenbrock}

Wissenschaftliche Erkenntnisse zur alter(n)sgerechten Arbeitsgestaltung werden nur ausgeschöpft, wenn sie Eingang in die betriebliche Praxis finden. Daher ist eine Verzahnung dieser beiden Bereiche von großer Bedeutung. Auch wenn sie in den vergangenen Jahren z.B. mithilfe von Fördermitteln der Bundes- und Landesregierungen oder Sozialpartnern gestärkt wurde, ist ein Durchdringen weiterhin noch nicht zu beobachten. Dies zu ändern und wissenschaftliche Erkenntnisse in eine praxisnahe Sprache und Instrumente zu ,übersetzen', wird weiterhin eine zentrale Aufgabe im Themenfeld sein, wobei Gewerkschaften und Berufsgenossenschaften hier unterstützend, für eine Umsetzung präventiver Arbeitsgestaltung insbesondere in KMU wirken können (s. Beitrag Langhoff und Anlauft \& Feldes in diesem Band). Die konsequente Umsetzung der Gefährdungsbeurteilung ist ein wichtiger Schritt für Unternehmen, Arbeitsbedingungen näher zu betrachten und Gestaltungspotenziale zu erkennen und auszuschöpfen. Da das Risiko für Erwerbsminderung nachweislich abhängig ist von den Arbeitsanforderungen und der Expositionszeit über den Erwerbsverlauf (z.B. Berufsjahre, Vollzeit/ Teilzeit; Mika, 2013), erleben nicht nur die Beschäftigten selbst, sondern auch die Unternehmen die Vorteile einer alter(n)sgerechten Arbeitsgestaltung. Die Wettbewerbsfähigkeit wird gestärkt, in dem Unternehmen rechtzeitig die Ressourcen und Potenziale ihrer Mitarbeiterinnen und Mitarbeiter entwickeln, ausbauen und Arbeit alternsgerecht gestalten und damit die Arbeits- und Beschäftigungsfähigkeit fördern.

\section{Fazit}

Eine alterns- und altersgerechte Arbeitsgestaltung ist ein zentraler Ansatz, um die Arbeitsfähigkeit alternder Belegschaften über die Lebensspanne zu fördern. Die Bedeutung gut gestalteter Arbeit nimmt dabei mit dem Alter zu. Weitere Bemühungen, insbesondere die Verzahnung von Wissenschaft und Praxis sowie die Integration von interanationaler Forschung und nationaler Anwendung sind notwendig, um die Umsetzung der Empfehlungen zu ermöglichen und zu fördern.

Konkret bedarf es einer Expertise in Unternehmen, allgemeine Erkenntnisse auf die spezifische Tätigkeitsebene zu transferieren. Die Gefährdungsbeurteilung kann dazu einen wesentlichen Beitrag leisten, da mit diesem Instrument ein Veränderungsprozess im Unternehmen, in Abteilungen und Teams angestoßen wird, der es ermöglicht, das Thema „Altern“ und der lebensspannenbezogenen Arbeitsgestaltung einzubeziehen.

Viele Aktivitäten in Forschung und betrieblicher Praxis in Unternehmen und Verwaltungen haben das Thema Arbeitsgestaltung im Kontext 
des immer stärker spürbaren demografischen Wandels in den vergangenen Jahren gestärkt und viele neue Erkenntnisse geliefert. Die digitale Transformation verändert die Arbeitswelt jedoch aktuell deutlicher und ist damit für viele Unternehmen ein bedeutenderes Thema. Hier liegt jedoch auch für die alter(n)sgerechte Arbeitsgestaltung ein Potenzial, die mit der Digitalisierung verbundenen Herausforderungen zu integrieren. Räumliche Mobilität, atypische Arbeitsverhältnisse, diskontinuierliche Berufsverläufe oder eine stärker werdende zeitliche Flexibilisierung sind weitere Aspekte der Arbeitsgestaltung. Wie reagieren Beschäftigte im Verlauf der Lebensspanne auf die Veränderungen? Wie können Unternehmen und Führungskräfte diese Veränderungen unterstützend begleiten, um die Regulation der Beschäftigten zu fördern und damit ihre Gesundheit und Leistungsfähigkeit zu stärken?

Die von Prof. Wachtler vor 20 Jahren angemahnten „tiefgreifenden Restrukturierungs- und Umgestaltungsprozesse" im Kontext des demografischen Wandels (s. oben; von Rothkirch, 2000, S. 49) sind bislang nur partiell verwirklicht worden. Zwar wurden viele Erkenntnisse gewonnen und einige Infrastrukturen zur Wissensweitergabe, wenn auch zumeist nur projektmäßig finanziert, geschaffen, z.B. durch die Initiative Neue Qualität der Arbeit (INQA). Der Weg vom Wissen hin in die Umsetzung ist jedoch z.B. in vielen KMUs weit. Dennoch scheint zunehmend erkannt zu werden, dass sich schablonenartige Maßnahmen der Arbeitsgestaltung im Sinne von ,one size fits all' nicht dazu eignen, auf besondere Herausforderungen, wie alternde Belegschaften oder digitale Transformationen zu reagieren. Vielmehr bedarf es organisierter Prozesse, wie z.B. Gefährdungsbeurteilungen oder Betriebsvereinbarungen, die einen verbindlichen Rahmen für Gestaltungsempfehlungen geben. Die Feinjustierung sollte jedoch individuell erfolgen. So ist es Aufgabe z.B. von Führungskräften, über Maßnahmen situativ und bedarfsorientiert zu entscheiden und deren Umsetzung zu steuern - und dazu bedarf es nicht nur der Expertise, sondern auch der Motivation auf Ebene der Unternehmensleitung und operativer Führungskräfte.

\section{Literatur}

Bakker, A. B., \& Demerouti, E. (2007). The job demands-resources model: State of the art. Journal of Managerial Psychology, 22, 309-328.

Baltes, P. B. (1987). Theoretical propositions of life-span developmental psychology: On the dynamics between growth and decline. Developmental psychology, 23(5), 611-626. 


\section{Müblenbrock}

Baltes, P. B., \& Baltes, M. M. (1990). Psychological perspectives on successful aging: The model of selective optimization with compensation. In: P. B. Baltes \& M. M. Baltes (Hrsg.), Successful aging: Perspectives from the behavioral sciences (S. 1-34). New York: Cambridge University Press.

BAuA Bundesanstalt für Arbeitsschutz und Arbeitsmedizin (2016). Ratgeber zur Gefährdungsbeurteilung. Handbuch für Arbeitsschutzfachleute. Dortmund: Bundesanstalt für Arbeitsschutz und Arbeitsmedizin.

BAuA Bundesanstalt für Arbeitsschutz und Arbeitsmedizin (Hrsg.) (2017). Alternsund altersgerechte Arbeitsgestaltung. Grundlagen und Handlungsfelder für die Praxis. https:/www.baua.de/DE/Angebote/Publikationen/Praxis/Arbeitsgestaltu ng.pdf. Abrufdatum: 14.07.2020.

Beck, D., et al. (2016). Arbeitsschutz in der Praxis: Empfehlungen zur Umsetzung der Gefährdungsbeurteilung psychischer Belastung. https:/www.gda-psyche.de/ SharedDocs/Downloads/DE/empfehlungen-zur-umsetzung-der-gefaehrdungsbeu rteilung-psychischer-belastung.html. Abrufdatum: 06.07.2020.

Bögel, J., \& Frerichs, F. (2011). Betriebliches Alters- und Alternsmanagement: Handlungsfelder, Maßnahmen und Gestaltungsanforderungen. BOD GmbH.

Brenscheidt, F. (2017). Gestaltung der Arbeitszeit aus Sicht des Arbeits- und Gesundheitsschutzes. In: G. Richter, C. Hecker \& A. Hinz (Hrsg.), Produktionsarbeit in Deutschland - mit alternden Belegschaften (S.96-108). Berlin: Erich Schmidt.

Carstensen, L. L. (2006). The influence of a sense of time on human development. Science, 312(5782), 1913-1915.

de Lange, A. H., et al. (2006). Age as a factor in the relation between work and mental health: results from the longitudinal TAS survey. In: J. Houdmont \& S. McIntyre (Hrsg.), Occupational Health Psychology: European Perspectives on Research, Education and Practice, Vol. 1 (S. 21-45). Maia: ISMAI Publications.

Gerst, D. (2013). Produktionssysteme alternsgerecht gestalten. Arbeitshilfe für betriebliche Interessenvertretungen. Industriegewerkschaft Metall (Hrsg.). Frankfurt a. M.: IG Metall.

Godde, B., Oltmanns, J., Richter, G., Voelcker-Rehage, C., \& Staudinger, U. M. (2019). Arbeits- und Karrieregestaltung zur Förderung der kognitiven Entwicklung im Erwachsenen- und höheren Alter. In: M. Falkenstein \& C. Kardys (Hrsg.), Arbeit, Kognition und Alter. Wissenschaftliche Erkenntnisse für die Praxis. Stuttgart: Kohlhammer.

Hacker, W. (1998). Allgemeine Arbeitspsychologie: Psychische Regulation von Arbeitstätigkeiten. Bern: Huber.

Hasselhorn, H. M. (2015). Arbeit, Alter und Gesundheit. In: J. v. Stein, I. Rothe, \& R. Schlegel (Hrsg.), Gesundheitsmanagement und Krankheit im Arbeitsverhältnis (S. 240-250). München: Beck.

Heckhausen, J., Wrosch, C., \& Schulz, R. (2010). A motivational theory of life-span development. Psychological Review, 117(1), 32-60. 
Hertel, G., \& Zacher, H. (2018). Managing the aging workforce. In D. S. Ones, N. Anderson, C. Viswesvaran, \& H. K. Sinangil (Eds.). The SAGE Handbook of Industrial, Work, \& Organizational Psychology, 2nd Edition, Vol. 3 (pp. 396428). Thousand Oakes, CA: Sage.

Ilmarinen, J. (2017). Der Hausbau in der Praxis: Neue Ergebnisse aus internationalen Betriebsprojekten, Vortrag auf WAI Netzwerkkonferenz am 07.03.2017 in Berlin.

Jungmann, F., Bilinska, P., \& Wegge, J. (2014). Alter(n)sgerechte Führung. Trends der psychologischen Führungsforschung: Neue Konzepte, Methoden und Erkenntnisse, 27, 467-479.

Keil, M., Weiler, S., \& Feiner, M. (2017). Die ganzheitliche Ergonomiestrategie bei Audi am Praxisbeispiel Lärm und Beleuchtung. In: G. Richter, C. Hecker, \& A. Hinz (Hrsg.), Produktionsarbeit in Deutschland - mit alternden Belegschaften (S. 109-118). Berlin: Erich Schmidt.

Mika, T. (2013). Risiken für eine Erwerbsminderung bei unterschiedlichen Berufsgruppen. Bundesgesundheitsblatt-Gesundheitsforschung-Gesundheitsschutz, 56(3), 391-398.

Mühlenbrock, I., \& Hüffmeier, J. (2020). Differential Work Design for Different Age Groups? Zeitschrift für Arbeits- und Organisationspsychologie, 64(3), 171195.

Oldham, G. R., \& Fried, Y. (2016). Job design research and theory: Past, present and future. Organizational Behavior and Human Decision Processes, 136, 20-35.

Oltmanns J., et al. (2017a). Don't Lose Your Brain at Work - The Role of Recurrent Novelty at Work in Cognitive and Brain Aging. Frontiers in psychology, 8(117).

Oltmanns J., Richter G., Godde B., \& Staudinger, U. M. (2017b). Gesund altern am Band: Karrieredesign für Produktionsmitarbeiter in der Industrie. In: G. Richter, C. Hecker, \& A. Hinz (Hrsg.), Produktionsarbeit in Deutschland - mit alternden Belegschaften (S. 59-84). Berlin: Erich Schmidt.

Rothkirch, C. v. (2000). Altern und Arbeit: Herausforderung für Wirtschaft und Gesellschaft. Berlin: Edition Sigma.

Rudolph, C. W., Zacher, H., \& Baltes, B. B. (2019). Looking forward: A new agenda for studying work across the lifespan. In: B. B. Baltes, C. W. Rudolph, \& H. Zacher (Hrsg.), Work across the lifespan (S.605-623). Cambridge: Elsevier Academic Press.

Scheibe, S., \& Zacher, H. (2013). A lifespan perspective on emotion regulation, stress, and wellbeing in the workplace. In: P. L. Perrewé, J. Halbesleben, \& C. C. Rosen (Hrsg.), The role of emotion and emotion regulation in job stress and well being. Research in occupational stress and well-being, Vol. 11 (S. 163-193). Bingley: Emerald.

Schlittmeyer, S. (2017). Lärm - Erkenntnisse zu Schwerhörigkeit und weiteren Lärmwirkungen aus wissenschaftlicher Sicht. In: G. Richter, C. Hecker, \& A. Hinz (Hrsg.), Produktionsarbeit in Deutschland - mit alternden Belegschaften (S. 167-197). Berlin: Erich Schmidt. 


\section{Müblenbrock}

Soestmeyer, G. (2017). Sehen im Alter. In: G. Richter, C. Hecker \& A. Hinz (Hrsg.), Produktionsarbeit in Deutschland-mit alternden Belegschaften (S. 184-198). Berlin: Erich Schmidt.

Sonntag, K. \& Seiferling, N. (2017). Alters- und berufsbegleitende Veränderungen des Leistungsprofils - Voraussetzungen und Limitationen für gesundes Altern in der Produktion. In: G. Richter, C. Hecker \& A. Hinz (Hrsg.), Produktionsarbeit in Deutschland - mit alternden Belegschaften (S. 274-293). Berlin: Erich Schmidt.

Truxillo, D. M., Cadiz, D. M., \& Hammer, L. B. (2015). Supporting the aging workforce: A review and recommendations for workplace intervention research. Annu. Rev. Organ. Psychol. Organ. Behav., 2, 351-81

Wendsche, J., \& Lohmann-Haislah, A. (2018). Arbeitspausen gesundheits- und leistungsförderlich gestalten. Göttingen: Hogrefe.

Wong, C. M., \& Tetrick, L. E. (2017). Job crafting: Older workers' mechanism for maintaining person-job fit. Frontiers in psychology, 8(1548).

Zacher, H., \& Schmitt, A. (2016). Work characteristics and occupational wellbeing: the role of age. Frontiers in psychology, 7(1411).

Zacher, H., Hacker, W., \& Frese, M. (2016). Action regulation across the adult lifespan (ARAL): A metatheory of work and aging. Work, Aging and Retirement, 2(3), 286-306.

\section{Referenzen}

1 Die nachfolgenden Erläuterungen zu den vier Merkmalsbereichen sind aus BAuA (2017) mit Quellenergänzungen entnommen. 